\title{
An investigation of 3D images of the simultaneous-lightness- contrast illusion using a virtual-reality technique
}

\author{
Galina Ya. Menshikova \\ Lomonosov Moscow State University, Moscow, Russia
}

\begin{abstract}
This article investigates the problem of lightness perception. To clarify the role of depth in lightness perception two current models-the albedo hypothesis and the coplanar-ratio hypothesis-are discussed. To compare them the strength of the simultaneous-lightnesscontrast (SLC) illusion was investigated as a function of three-dimensional (3D) configurations of the test and background squares. In accordance with both hypotheses the changes in the depth arrangements of the test and background squares should result in changes in the illusory effect. However, the reasons for and the directions of these changes should be different. Five different types of 3D configurations were created in which the test squares were tilted at different angles to the background squares. A virtual-reality technique was used to present stereo pairs of different 3D configurations. Thirty-seven observers took part in the experiment. The method of constant stimuli was used to obtain psychometric functions. The displacements of these functions for 3D configurations in comparison with the $2 \mathrm{D}$ configuration allowed the estimation of illusion strength. The analysis of individual values of illusion strength revealed two groups of subjects. For the first group (38\% of all participants) the strength changed insignificantly depending on the $3 \mathrm{D}$ configurations. For the second group (62\% of all participants) significant differences were obtained for those configurations in which the test and background squares were perceived as differently illuminated. The changes in the SLC illusion strength for the second group were consistent with predictions made by the albedo hypothesis. Thus, it seems that the perceived illumination of a surface should be considered the main parameter for lightness estimations in 3D scenes.
\end{abstract}

Keywords: perception, visual illusions, lightness, perceived illumination, albedo hypothesis, coplanar-ratio hypothesis, virtual-reality technology

The illusion of simultaneous lightness contrast (SLC) has been actively investigated for 180 years. In the illusion (Figure 1) two identical gray squares located on lightgray and dark-gray backgrounds are perceived as dark-gray and light-gray respectively.

The problem of the SLC illusion is tightly connected with the problem of lightness perception. Many authors have considered the SLC illusion as a demonstration of their theoretical assumptions about the process of lightness perception. 


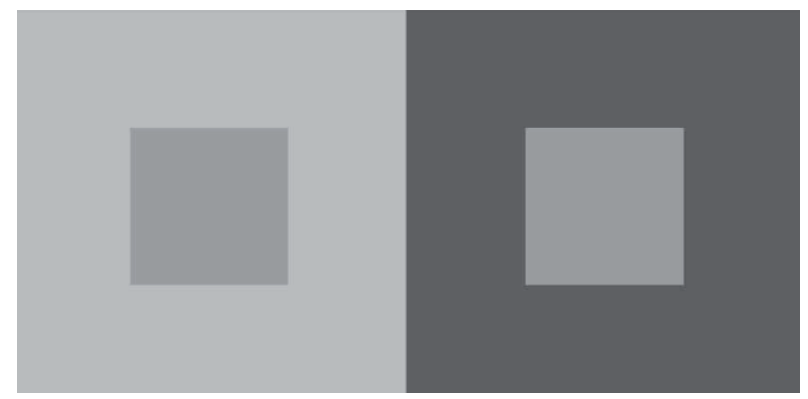

Figure 1. The SLC illusion

Let us preliminarily introduce some terms that will be used in this article. All surfaces absorb a certain amount of light and reflect the rest. The intensity of light falling on a surface is called the illumination. Luminance refers to the intensity of light reflected by a surface and projected onto the retina. The percentage of light reflected by a surface is called reflectance. For example, white surfaces reflect about $90 \%$ of light, while black surfaces reflect about $3 \%$ of it. So the ratio of the intensities of light reflected from white and black surfaces is 30:1. All surfaces are tilted relative to the subject and to the light source. Surfaces that are located in the same plane or in parallel planes are called coplanar. The perceived reflectance is referred to as lightness. The intensity of light perceived by the subject is called the perceived illumination.

Luminance is considered the proximal stimulus for lightness estimations. The problem of lightness perception lies in the fact that luminance depends not only on the surface's reflectance but also on other parameters of the external environment: the illumination of a surface and its slant. The surfaces around us are illuminated differently (some are shadowed, others are strongly illuminated) and are tilted differently in space. In special cases the light intensity coming from shaded white surfaces is less than the light intensity reflected from illuminated black surfaces. So a question arises about the mechanisms that the visual system uses for lightness estimations.

To solve the problem of lightness perception many hypotheses have been suggested. Let us consider some of those that are frequently debated. The first one assumes that the ratio of the luminance of the test surface to the luminance of other surfaces determines this process. Using these ratios it is possible to estimate the relative reflectance of all surfaces that are equally illuminated. Because in natural scenes objects lying in the same surface are as a rule illuminated uniformly, it was assumed (Gilchrist et al., 1999) that the luminance ratio of coplanar surfaces was a basic stimulus for lightness perception. A second hypothesis claims that the perceived illumination of a surface plays an important role in lightness estimations. This hypothesis was formulated by H. von Helmholtz (1867) and then was reformulated as the albedo hypothesis in contemporary theories of lightness constancy (Bergstrom, 1977; Kozaki \& Noguchi, 1976; Logvinenko \& Menshikova, 1994; Menshikova \& Lunyakova, 1994). It supposes that the lightness of the surface and its perceived illumination are coupled in the visual image. In accordance with the rule of their relationship, an increase in perceived illumination should be ac- 
companied by a decrease in lightness at a constant value of surface luminance. A third hypothesis emphasizes the role of local contrasts for lightness estimation. The idea was proposed by E. Hering (Hering , 1964) and has now been developed in a number of contemporary studies (Blakeslee \& McCourt, 1999; Blakeslee, Pasieka, \& McCourt, 2005; Grossberg \& Howe, 2003). A fourth approach for lightness perception considers the role of contour junctions-that is, the places where several surfaces having different degrees of luminance are contiguous to each other (Anderson, 1997; Ross \& Pessoa, 2000; Todorovic, 1997).

Let us consider the first two hypotheses in detail as they are the most discussed in the modern literature. The first one assumes that surface lightness can be evaluated through the ratio of its luminance to the luminance of other surfaces in the scene (Land \& McCann, 1971; Wallach, 1963). This procedure allows the relative reflectance of all surfaces to be computed. To estimate the absolute reflectance the anchoring rule is applied in the visual system. This means that one of the relative reflectance values anchors to some absolute value: for example, the most luminous object is assumed to be white (Gilchrist et al., 1999; Land \& McCann, 1971; Wallach, 1976). Thus, the lightness of each object can be determined. However, the described model of lightness estimations works when all surfaces are equally illuminated and there is only one light source in the scene. Otherwise, when there are differently illuminated surfaces, the luminance ratios depend on the intensity relationships of the strongly illuminated and the shadowed surfaces. It is assumed that the lightness estimation in complex scenes is accomplished in two stages: first the groups of coplanar surfaces are picked out, and then lightness is estimated in accordance with the anchoring rule for each group. The anchoring rules "work" in the range of local and global frameworks simultaneously. Local frameworks are used to estimate the luminance ratio of the test patch and adjacent background patches of a surface. Global frameworks are used for estimating the luminance ratio of the test patch and distant patches of a surface. A general lightness estimation is calculated as average lightness through local and global frameworks. This hypothesis was called the coplanar-ratio hypothesis (Gilchrist, 1977), and the visual cues of grouping were called the coplanarity cues. The important role of these cues in lightness perception was shown in a number of studies (Taya, Ehrenstein, \& Cavonius, 1995; Wishart, Frisby, \& Buckley, 1997).

The alternative hypothesis of lightness perception (the albedo hypothesis) argues for the strong effect of the perceived illumination on lightness estimation. The albedo hypothesis asserts that if the value of perceived illumination increases, lightness is underestimated by the subject, and vice versa: surface lightness is overestimated with a decrease in the value of perceived illumination. For example, the explanation of the SLC illusion (Figure 1) proposed by Helmholtz assumed that the surfaces of the light-gray and dark-gray backgrounds should be perceived as strongly illuminated/shadowed surfaces so this hypothesis would result in underestimation/overestimation of the central squares lightness. The basic problem with this hypothesis lies in the fact that it is necessary to explain the mechanisms for the estimation of perceived illumination. Unfortunately, no neuron mechanisms for illumination estimation were found, so the mechanism for the illumination estimation in the visual system and for the following lightness estimation remains unclear. In spite of these difficulties, results were obtained that confirmed 
the influence of the perceived illumination on lightness perception (Knill \& Kersten, 1991; Logvinenko \& Menshikova, 1994; Menshikova \& Lunyakova, 1994; Menshikova \& Nechaeva, 2011; Williams, McCoy, \& Purves, 1998).

In a number of studies the influence of depth on lightness perception was investigated to test two of the theoretical hypotheses mentioned above. The main idea behind these studies was to change the coplanarity cues by manipulations of $3 \mathrm{D}$ positions of the test surfaces relative to the background surfaces. In accordance with the coplanar-ratio hypothesis these manipulations would result in a change in lightness perception. Some works studying the influence of depth on lightness estimations (Kardos, 1934; Wolff, 1933) confirmed these predictions. In one of them (Wolff, 1933) two test surfaces with the same reflectance were arranged against dark and light backgrounds. When test surfaces were coplanar to their backgrounds, the SLC illusion appeared, and the two tests looked different. However, when the test surfaces were moved away from the backgrounds (that is, they were not coplanar), the strength of the SLC illusion decreased. These results showed that the relationship between the test surfaces and the background surfaces occurred only when they were coplanar. Later works also tested the coplanar-ratio hypothesis. The influence of depth cues on lightness perception was investigated using stereo images (Coren, 1969). The observer saw a black cross hanging over a white background. The test gray square was arranged next to the cross and was perceived as being located, in one case, in the plane of the cross, and, in another, in the plane of the background. The observer was asked to estimate the lightness of the test square. Results showed that the test square, perceived in the plane of the black cross, was estimated to be $7 \%$ lighter. However, that effect was rather weak. To test the influence of depth cues on lightness perception the study was carried out using the Gelb effect (Gogel \& Mershon, 1969). A large, black, strongly illuminated disk hanging over the black unlit background was perceived as white or light gray (the Gelb effect). When a small white disk was attached to the black disk, the Gelb effect decreased-the black disk was perceived as black. However, when the white disk was moved away from the black one (that is, when it was not coplanar), the Gelb effect increased again. Conditions of monocular/binocular viewing were used to investigate the influence of depth cues in lightness perception (Gilchrist, 1977). When the display was viewed monocularly, the test disk was perceived as being coplanar to a dark-gray surface, whereas when the display was viewed binocularly, the test disk seemed coplanar to a light-gray surface. As a result, the shift in lightness estimations in the two conditions was 4.5 Munsell units. The abovementioned studies were in good agreement with the coplanar-ratio hypothesis. Other studies investigating the role of depth cues did not reveal or determine the very weak influence of coplanar ratios on lightness estimations (Dalby, Saillant, \& Wooten, 1995; Epstein, 1961; Flock \& Freedberg, 1970; Julesz, 1971; Zaidi, Spehar, \& Shy, 1997).

A number of rules were given for applying the coplanar-ratio hypothesis correctly (Gilchrist, 1980). First, the retinal-luminance ratios of the compared surfaces should remain constant. Second, to change coplanar ratios it is necessary to create two different experimental conditions, A and B. Under the A condition the test surface should belong to one background, and under the B condition it should belong to another background. It was shown that when the test surface was not coplanar 
to the background surface, the coplanar-ratio hypothesis was not available, and as a result lightness estimations became worse (Gilchrist, 1980). Third, the coplanar ratio hypothesis "works" only when the luminance ratios are more than 30:1. This limitation made it possible to explain why the influence of depth cues on lightness was not revealed in many studies.

These studies provided contradictory results for the role of depth cues on lightness perception. Therefore, the question of choosing one of the alternative hypotheses remained unanswered. One of the problems of choosing an appropriate theoretical approach was connected with the fact that in many studies the experimental conditions were set up in such a way that changes in luminance ratios were accompanied by changes in perceived illumination. An attempt was made to test the two hypotheses under conditions of separate, independent changes in luminance ratios and perceived illumination (Howe, 2006). The results were in better agreement with the albedo hypothesis.

In our study the coplanar-ratio hypothesis and the albedo hypothesis were tested under conditions of independent changes in luminance ratios and perceived illumination by exploring the SLC illusion. The strength of the SLC illusion was investigated as a function of $3 \mathrm{D}$ configurations of the test and background squares. In accordance with both hypotheses the changes in depth arrangements of the test and background squares should result in changes in the illusory effect. However, the reasons for and the directions of these changes should be different for each hypothesis. In line with the albedo hypothesis, changes in illusion strength should be connected with the fact that changes in the space arrangement of the test and background squares would result in changes of perceived illumination and, in turn, would result in changes of lightness (Logvinenko \& Menshikova, 1994; Menshikova \& Lunyakova, 1994). Consequently, by tilting test squares toward/down from the hypothetical overhead light source, it is possible to increase/decrease perceived illumination and therefore to decrease/increase lightness. Changes in the lightness of the test squares should result in illusion strength. If the test squares are tilted differently relative to the hypothetical overhead light source, then perceived illumination should be stronger for one of them and weaker for the other, and as a result the illusion strength should change. But if the test squares are tilted equally relative to the hypothetical light source, the illusion strength should remain constant. In line with the coplanar-ratio hypothesis lightness estimations are determined by the anchoring rule, which depends on coplanarity cues available in the scene. Reducing these cues should result in a decrease in illusion strength. To reduce them the classical 2D illusion was transformed into different 3D configurations. There were two types of transformations. For the first type, the test squares were tilted relative to the background squares (the break of local frameworks); for the second, the background squares were tilted relative to each other (the break of global frameworks). Both types of transformations should result in the reduction of coplanarity cues and then in the reduction of illusion strength for all 3D configurations independently of the spatial arrangement of the test and background squares. However, the reduction should be expressed less for the second transformation type than for the first because the break of local frameworks is more significant than the break of global frameworks for lightness perception. 


\section{Method}

\section{Participants}

Thirty seven observers (12 males and 25 females; age range 17-30) with normal or corrected-to-normal vision were tested. All the observers were unaware of the purpose of the experiment.

\section{Apparatus}

Virtual-reality technology was used to present 2D-3D configurations of the SLC illusion. Advantages of and perspectives on this technology in experimental psychology, cognitive psychology, and psychophysiology have been thoroughly described and analyzed in the Russian and international literature (Riva, 2005; Yee, 2007; Zinchenko, Menshikova, Bayakovsky, Chernorizov, \& Voiskounsky, 2010; Zinchenko, Menshikova, Chernorizov, \& Voiskounsky, 2011). Participants observed 2D-3D images of SLC illusions using the head-mounted-display (HMD) technique eMagin Z800 3D Visor. ${ }^{*}$ The HMD displays were arranged at a distance of $2.5 \mathrm{~cm}$ from observers' eyes. The angular sizes of the test and background squares were $2^{\circ} \times 2^{\circ}$ and $6^{\circ} \times 6^{\circ}$ respectively. Maximum luminance was equal to $333 \mathrm{~cd} / \mathrm{m}^{2}$. The luminance ratio was 52:1.

\section{Stimuli}

The articulated variant of the SLC illusion (Figure 2) was used as the basic pattern because its illusory effect was stronger than that of the original (Adelson, 2000; Gilchrist et al., 1999; Soranzo, Lugrin, \& Cavazza, 2011).

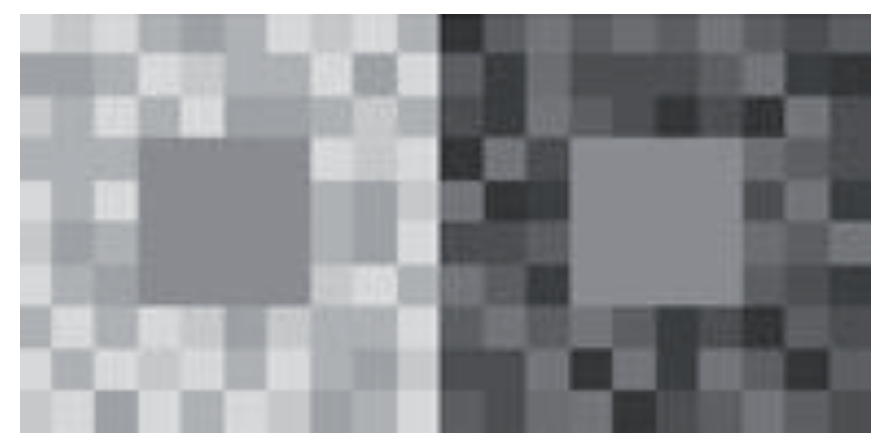

Figure 2. Articulated variant of the SLC illusion

Different 3D configurations of the SLC illusion were formed by the creation of stereo pairs with the use of Photoshop CS2 9.0. The value of disparity was constant and equal to $10 \mathrm{pxl}$ for all stereo pairs.

Five different types of 2D-3D configurations of the test and background squares were created. They were arranged against the frontal random-point surface. The types were as follows: type 1-2D articulated variant of the SLC illusion (Figure 2);

* The work was done on equipment acquired by the Lomonosov Moscow State University Program of Development. 
type 2-the central squares were moved out of the background squares and were parallel to them (Figure 3.2); type 3-the central squares were tilted at the same angles to their backgrounds (Figure 3.3); type $4 \mathrm{a}$ and $4 \mathrm{~b}$-the central squares were tilted at different angles to their backgrounds: the left one was tilted toward the hypothetical overhead light source, and the right one was tilted downward from it (Figure 3.4a), and vice versa (Figure 3.4b); type 5a and 5b-the background squares were tilted at different angles to the frontal random-point surface in a similar way as for types $4 \mathrm{a}, 4 \mathrm{~b}$.

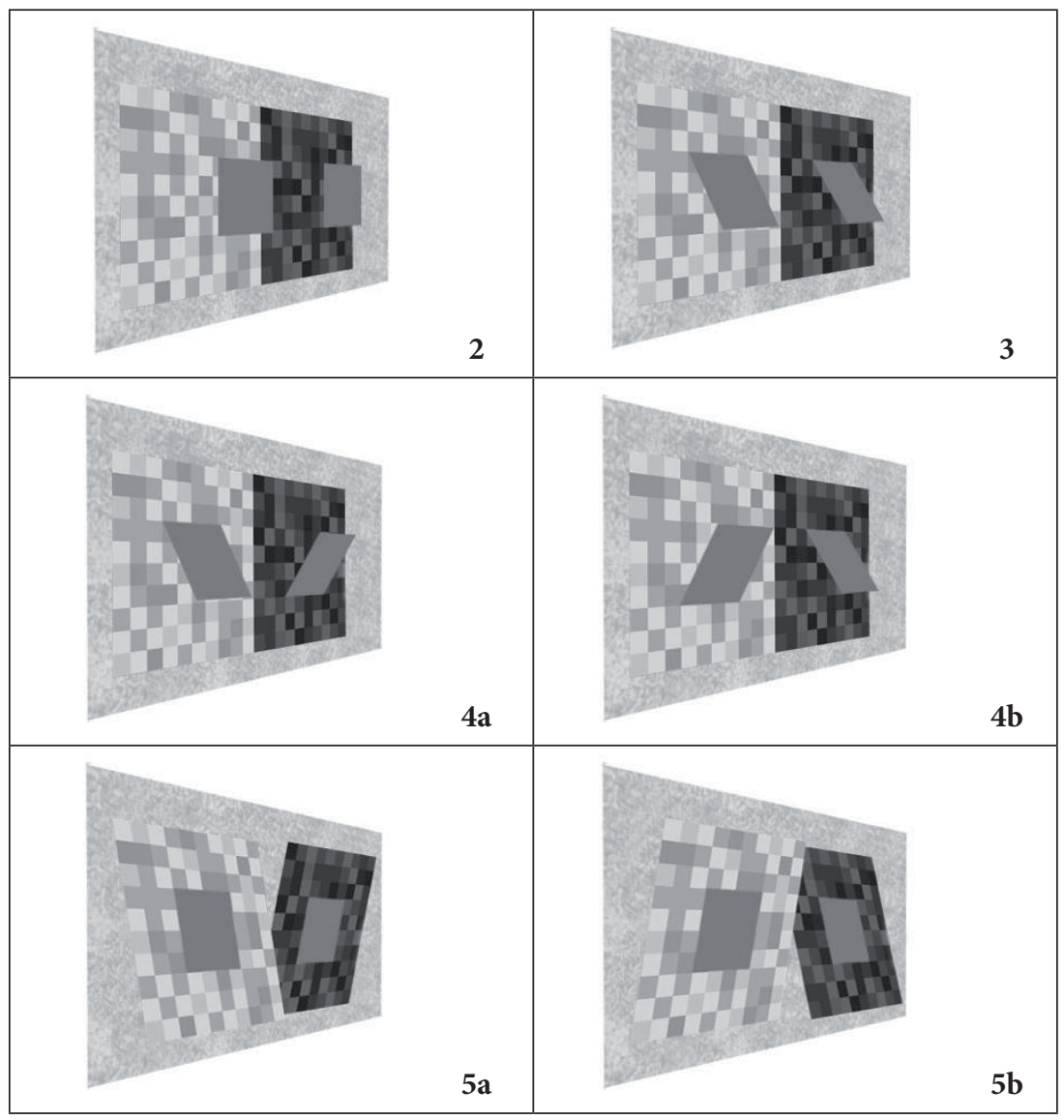

Figure 3. Types of 3D configurations of the SLC illusion

The method of constant stimuli was used to estimate the strength of the SLC illusion. The initial brightness of test squares was equal to $44 \%$ of black shade according to the Grayscale. Six variable stimuli were created for every 2D-3D configuration, for which the value of brightness for the test square lying on the light-gray background was constant and the value of brightness for the test square lying on the dark-gray background decreased from $44 \%$ to $70 \%$ of black shade with a step of $5 \%$. Thus, 42 stereo pairs were created: seven 2D-3D configurations, each having six variable stimuli. 


\section{Procedure and Plan}

The stimuli sequence was created in MediaLab v2008. 1.33. Each of the 42 stereo pairs was repeated 10 times. The sequence of stimuli presentation was completely randomized. The participant's ability to see $3 \mathrm{D}$ configurations was tested at the beginning of the experiment. Then 420 stereo pairs were presented. The time for presentation of each stimulus was $5 \mathrm{~s}$. The screen with possible answers appeared after each presentation. In every trial the participant was asked to choose the lighter of the two central squares.

\section{Results and Discussion}

Psychometric functions for 2D and six different 3D configurations were obtained and were used to evaluate the strength of the SLC illusion for each participant and each 3D configuration. The illusion strength (IS) was calculated as IS $=\left(\mathrm{P}_{3 \mathrm{D}} /{ }_{2 \mathrm{D}}-1\right) \times 100 \%$, where $\mathrm{P}_{3 \mathrm{D}}$ and $\mathrm{P}_{2 \mathrm{D}}-50 \%$ probability of answers "lighter" for $3 \mathrm{D}$ and $2 \mathrm{D}$ configurations respectively. Analysis of individual values of the SLC illusion strength revealed two groups of subjects. The values of the illusion strength were averaged for each group separately. The results are shown in Figure 4.

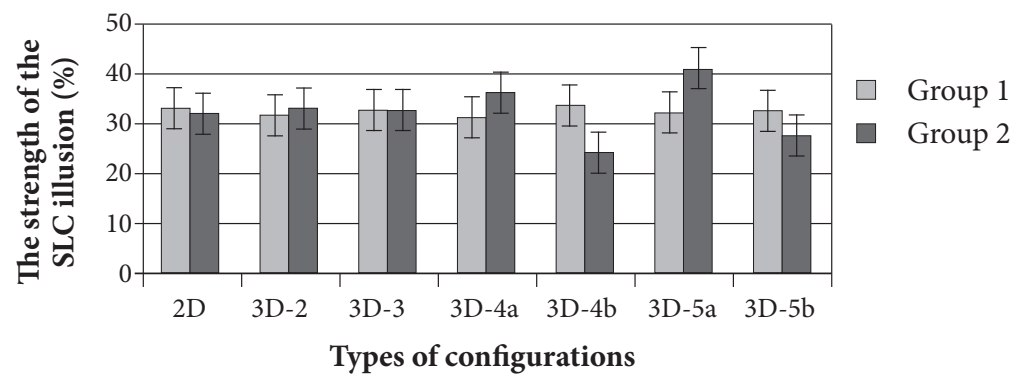

Figure 4. The strength of the SLC illusion as a function of 2D-3D configurations

For the first group (38\% of all the participants) the strength changed insignificantly depending on $2 \mathrm{D}-3 \mathrm{D}$ configurations; significant difference $t(72)=1.86$, $p=0.05$. For the second group (62\% of all the participants) significant differences were revealed for types $4 \mathrm{a}-4 \mathrm{~b}(t(72)=2.77, p \square 0.01)$, and also for types $5 \mathrm{a}-5 \mathrm{~b}$ $(t(72)=2.82, p \square 0.01)$ of $3 \mathrm{D}$ configurations. The results showed an increase of the illusion effect for types 3D-4a and 3D-5a and, on the contrary, a decrease for types $3 \mathrm{D}-4 \mathrm{~b}$ and $3 \mathrm{D}-5 \mathrm{~b}$.

The data were analyzed in accordance with the albedo hypothesis and the coplanar-ratio hypothesis. In line with the coplanar-ratio hypothesis the space separation of the test squares relative to the background squares should lead to a reduction of coplanarity cues and, as a result, to a reduction of illusion strength (IS). The value of the reduction should be different for different types of 3D configurations. The break of local frameworks (configurations 3D-2, 3D-3, 3D-4a, and 3D-4b) should result in a stronger decrease of IS than the break of global frameworks (configurations 3D-5a and 3D-5b). As shown in Figure 4, the changes of IS did not confirm these predictions. For the first group there were no significant changes 
of IS at all. For the second group the decreases of the illusion effect were found for types $3 \mathrm{D}-4 \mathrm{~b}$ and $3 \mathrm{D}-5 \mathrm{~b}$, but they were not consistent with the coplanar-ratio hypothesis. According to the albedo hypothesis, changes in IS should occur only for those 3D configurations in which the test and background squares seemed differently illuminated. In the absence of cast and attached shadows the visual system should make "an unconscious inference" about the overhead light source. For configurations $2 \mathrm{D}, 3 \mathrm{D}-2$, and $3 \mathrm{D}-3$ the test squares were tilted equally relative to the overhead light source, so IS did not change. For the configuration 3D-4a the test square lying on the light-gray background was tilted toward the hypothetical light source, so it appeared darker in contrast to the test square lying on the dark-gray background, which was tilted downward from the hypothetical light source and so appeared lighter. This configuration resulted in an increase of IS values. Using the same inferences it is possible to explain the decrease of IS values for the $3 \mathrm{D}-4 \mathrm{~b}$ configuration. The changes of illusion strength for 3D-5a and 3D-5b may be interpreted similarly.

\section{Conclusion}

Two current models of lightness perception-the albedo hypothesis and the coplanar-ratio hypothesis-were tested to explain the results of the study. The changes in the SLC illusion strength for different 2D-3D configurations were consistent with predictions made by the albedo hypothesis. It seems that in $3 \mathrm{D}$ scenes the perceived illumination of a surface should be considered as the main parameter for lightness estimations.

\section{Acknowledgments}

This research was supported by the grant "Development of innovative methods for scientific, educational, and practical activities of psychologists with the application of virtual-reality technologies" within the framework of the federal program "Scientific and scientific-pedagogical personnel of innovative Russia" for 2009-2013 (State contract no. 8011).

\section{References}

Adelson, E. H. (2000). Lightness perception and lightness illusions. In M. S. Gazzaniga (Ed.), The new cognitive neurosciences (2nd ed., pp. 339-351). Cambridge, MA: MIT Press.

Anderson, B. L. (1997). A theory of illusory lightness and transparency in monocular and binocular images. Perception, 26, 419-453. PMid:9404492 doi: 10.1068/p260419

Bergstrom, S. S. (1977). Common and relative components of reflected light as information about the illumination, colour, and three-dimensional form of objects. Scandinavian Journal of Psychology, 18, 180-186. PMid:897594 doi: 10.1111/j.1467-9450.1977.tb00275.x

Blakeslee B., \& McCourt, M. E. (1999). A multiscale spatial filtering account of the White effect, simultaneous brightness contrast and grating induction. Vision Research, 39, 4361-4377. doi: 10.1016/S0042-6989(99)00119-4

Blakeslee, B., Pasieka, W., \& McCourt, M. E. (2005). Oriented multiscale spatial filtering and contrast normalization: A parsimonious model of brightness induction in a continuum 
of stimuli including White, Howe and simultaneous brightness contrast. Vision Research, 45(5), 607-615. PMid:15621178 doi: 10.1016/j.visres.2004.09.027

Anderson, B. L. (1997). A theory of illusory lightness and transparency in monocular and binocular images. Perception, 26, 419-453. doi: 10.1068/p260419

Bergstrom, S. S. (1977). Common and relative components of reflected light as information about the illumination, colour, and threedimensional form of objects. Scandinavian Journal of Psychology, 18, 180-186. doi: 10.1111/j.1467-9450.1977.tb00275.x

Blakeslee, B., \& McCourt, M. E. (1999). A multiscale spatial filtering account of the White effect, simultaneous brightness contrast and grating induction. Vision Research, 39, 4361-4377. doi: 10.1016/S0042-6989(99)00119-4

Blakeslee, B., Pasieka, W., \& McCourt, M. E. (2005). Oriented multiscale spatial filtering and contrast normalization: A parsimonious model of brightness induction in a continuum of stimuli including White, Howe and simultaneous brightness contrast. Vision Research, 45(5), 607-615. doi: 10.1016/j.visres.2004.09.027

Coren, S. (1969). Brightness contrast as a function of figure-ground relations. Journal of Experimental Psychology, 80, 517-524. doi: 10.1037/h0027435

Dalby, T. A., Saillant, M. L., \& Wooten, B. R. (1995). The relation of lightness and stereoscopic depth in a simple viewing situation. Perception \& Psychophysics, 57(3), 318-332. doi: 10.3758/BF03213057

Epstein, W. (1961). Phenomenal orientation and perceived achromatic color. Journal of Psychology, 52, 51-53. doi: 10.1080/00223980.1961.9916503

Flock, H. R., \& Freedberg, E. (1970). Perceived angle of incidence and achromatic surface color. Perception \& Psychophysics, 8, 251-256. doi: 10.3758/BF03210216

Gilchrist, A. L. (1977). Perceived lightness depends on perceived spatial arrangement. Science, 195(4274), 185-187. doi: 10.1126/science.831266

Gilchrist, A. L. (1980). When does perceived lightness depend on perceived spatial arrangement? Perception \& Psychophysics, 28(6), 526-538. doi: 10.3758/BF03198821

Gilchrist, A. L., Kossyfidis, C., Bonato, F., Agostini, T., Cataliotti, J., Li, X., Spehar, B., \& Annan, V. (1999). An anchoring theory of lightness perception. Psychological Review, 106(4), 795-834. doi: 10.1037/0033-295X.106.4.795

Grossberg, S., \& Howe, P. D. (2003). A laminar cortical model of stereopsis and three- dimensional surface perception. Vision Research, 43, 801-829. doi: 10.1016/S0042-6989(03)00011-7

Grossberg, S., \& Todorovic, D. (1988). Neural dynamics of 1-D and 2-D brightness perception: a unified model of classical and recent phenomena. Perception \& Psychophysics, 43, 241-277. doi: 10.3758/BF03207869

Gogel, W. C., \& Mershon, D. H. (1969). Depth adjacency and simultaneous contrast. Perception \& Psychophysics, 5, 13-17. doi: 10.3758/BF03210471

Helmholtz, H. von. (1867). Handbuch der physiologischen optik [Handbook of physiological optics]. Leipzig: Voss.

Hering, E. (1964). Outlines of a theory of the light sense (L. Hurvich \& D. Jameson, Trans.). Cambridge, MA: Harvard University Press. (Original work published 1874)

Howe, P. D. (2006). Testing the coplanar ratio hypothesis of lightness perception. Perception, 35(3), 291-301. doi: 10.1068/p5461

Julesz, B. (1971). Foundations of Cyclopean perception. Chicago: Chicago University Press.

Kardos, L. (1934). Ding und Schatten. Eine experimentelle Untersuchung über die Grundlagen des Farbensehens [Object and shadow. An experimental investigation of the fundamentals of color vision]. Zeitschrift fur Psychologie Erganzungsband, 23, 1-184.

Knill, D. C., \& Kersten, D. (1991). Apparent surface curvature affects lightness perception. Nature, 351(6323), 228-230. doi: 10.1038/351228a0 
Kozaki, A., \& Noguchi, K. (1976). The relationship between perceived surface-lightness and perceived illumination. Psychological Research, 39, 1-16. doi: 10.1007/BF00308942

Land, E. H., \& McCann, J. J. (1971). Lightness and retinex theory. Journal of the Optical Society of America, 61, 1-11 doi: 10.1364/JOSA.61.000001

Logvinenko, A., \& Menshikova, G. (1994). Trade-off between achromatic colour and perceived illumination as revealed by the use of pseudoscopic inversion of apparent depth. Perception, 23, 1007-1023. doi: 10.1068/p231007

Menshikova, G., \& Lunyakova, E. (1994). Relationship between achromatic color of a surface and its perceived illumination in the "wallpaper" illusion. Perception, 23, 17.

Menshikova, G., \& Nechaeva, A. (2011). Does the strength of simultaneous lightness contrast depend on the disparity cue? Perception, 40, 104.

Riva, G. (2005). Virtual reality in psychotherapy: Review. CyberPsychology \& Behavior: The Impact of the Internet, Multimedia and Virtual Reality on Behavior and Society, 8, 220-230. doi: $10.1089 / \mathrm{cpb} .2005 .8 .220$

Ross, W., \& Pessoa, L. (2000). Lightness from contrast: A selective integration model. Perception \& Psychophysics, 62, 1160-1181. doi: 10.3758/BF03212120

Soranzo, A., Lugrin, J-L., \& Cavazza M. (2011). Photometric relationships affect the simultaneous lightness contrast phenomenon in virtual reality. Perception, 40, 76.

Taya, R., Ehrenstein, W. H., \& Cavonius, C. R. (1995). Varying the strength of the Munker-White effect by stereoscopic viewing. Perception, 24(6), 685-694. doi: 10.1068/p240685

Todorovic, D. (1997). Lightness and junctions. Perception, 26, 379-394. doi: 10.1068/p260379

Wallach, H. (1963). The perception of neutral colours. Scientific American, 208, 107-116. doi: 10.1038/scientificamerican0163-107

Wallach, H. (1976). On perception. New York: Quadrangle/New York Times Book Co.

Williams, S. M., McCoy, A. N., \& Purves, D. (1998). The influence of depicted illumination on brightness. Proceedings of the National Academy of Sciences of the USA, 95, 13296-13300. doi: 10.1073/pnas.95.22.13296

Wishart, K. A., Frisby, J. P., \& Buckley, D. (1997). The role of 3-D surface slope in a lightness/ brightness effect. Vision Research, 37(4), 467-473. doi: 10.1016/S0042-6989(96)00104-6

Wolff, W. (1933). Uë ber die kontrasterregende Wirkung der transformierten Farben [Concerning the contrast-causing effect of transformed colors]. Psychologische Forschung, 18(1), 9097. doi: $10.1007 / \mathrm{BF} 02409628$

Yee, N. (2007). Psychological research in virtual worlds. Retrieved from http://bps-researchdigest. blogspot.com/2007/06/psychological-research-in-virtual.html

Zaidi, Q., Spehar, B., \& Shy, M. (1997). Induced effects of backgrounds and foregrounds in threedimensional configurations: The role of T-junctions. Perception, 26, 395-408. doi: 10.1068/ p260395

Zinchenko, Yu. P., Men'shikova, G. Ya., Bayakovsky, Yu. M., Chernorizov, A. M., \& Voiskounsky, A. E. (2010). Technologies of virtual reality in the context of world-wide and Russian psychology: Methodology, comparison with traditional methods, achievements and perspectives. Psychology in Russia: State of the Art, 3, 12-45. doi: 10.11621/pir.2010.0001

Zinchenko, Yu. P., Menshikova, G. Ya., Chernorizov, A. M., \& Voiskounsky, A. E. (2011). Technologies of virtual reality in psychology of sports of great advance: Theory, practice and perspectives. Psychology in Russia: State of the Art, 4, 129-154. doi: 10.11621/pir.2011.0008 